\title{
Redox Modulating Factors Affect Longevity Regulation in Rotifers
}

\author{
Lilla Macsai, MSc, ${ }^{1}$ Zita Olah, PhD, ${ }^{1}$ Ashley I. Bush, MD, PhD, DSc, ${ }^{2}$ \\ Bence Galik, MSc, ${ }^{3}$ Rita Onody, PhD, ${ }^{4}$ Janos Kalman, MD, PhD, DSc, ${ }^{1}$ and \\ Zsolt Datki, PhD ${ }^{1, \oplus}$
}

'Department of Psychiatry, Faculty of Medicine, Albert Szent-Gyorgyi Clinical Center, University of Szeged, Hungary. ${ }^{2}$ Oxidation Biology Unit, Florey Institute of Neuroscience and Mental Health, University of Melbourne, Parkville, Victoria, Australia. ${ }^{3}$ Bioinformatics and Scientific Computing, Vienna Biocenter Core Facilities, Austria. ${ }^{4}$ Department of Laboratory Medicine, Faculty of Medicine, Albert SzentGyorgyi Clinical Center, University of Szeged, Hungary.

Address correspondence to: Zsolt Datki, PhD, Department of Psychiatry, Faculty of Medicine, Albert Szent-Gyorgyi Clinical Center, University of Szeged, Hungary. E-mails: datkizsolt@gmail.com; datki.zsolt@med.u-szeged.hu

Received: January 22, 2018; Editorial Decision Date: August 15, 2018

Decision Editor: Rozalyn Anderson, PhD

\begin{abstract}
Rotifers are microinvertebrate models to study the phylogenetically based mechanisms of aging. Our study aimed to develop a physiological system with electron deprivation via a chemical electron carrier/acceptor pair together with extreme caloric restriction (ECR). Middle-aged Philodina acuticornis rotifers were treated with combinations of phenazine methosulfate (PMS, electron carrier) and 2,3-bis(2-methoxy-4nitro-5-sulfophenyl)-2H-tetrazolium-5-carboxanilide inner salt (XTT, electron acceptor) for a period of 72 hours under total food deprivation (preselection). The ability of XTT to be reduced was confirmed both in vitro (with NADH) and in vivo (with live rotifers). Subsequently, the respective electron acceptor alone at a lower dose was administered in combination with ECR for several months on preselected survivors. We found that the longevity of rotifers markedly increased (4x) after PMS/XTT/total food deprivation preselection followed by XTT/ECR treatment. Ascorbic acid in equivalent concentrations caused similar but less pronounced tendencies. The synergistic effect of chemical electron deprivation and ECR caused delayed aging and the development of an outstanding phenotype that we refer to as "super rotifers," characterized by increased longevity and retained reproductive ability compared with normal middle-aged individuals. The presented model provides new insights into the connection between redox modulation and age-related features in vivo.
\end{abstract}

Keywords: Antiaging, Caloric restriction, Life-span extension, Invertebrate, Bdelloid rotifer

Rotifers are widely used in the research fields of ecotoxicology and aging $(1,2)$. They are eutelic (grown by hypertrophy) and are promising scientific models due to their optimal culturing requirements $(3,4)$, short life span, and specific measurable phenotypic features and viability markers (5). Treatment with electron carriers and acceptors increases the life span of various organisms by increasing the cellular $\mathrm{NAD}^{+}: \mathrm{NADH}$ ratio (6,7). The 2,3-bis(2-methoxy-4-nitro-5-sulfophenyl)-2H-tetrazolium-5-carboxanilide inner salt (XTT) and phenazine methosulfate (PMS) molecules are suitable manipulators of cellular redox state (8). XTT has a positively charged quaternary tetrazole ring core containing four nitrogen atoms. It has a redox potential that is lower than the transmembrane potential, hence promoting the transfer of intracellular electrons to the extracellular space $(9,10)$. The sulfonate groups provide a net negative charge, promoting extracellular exclusion and water solubility (8). XTT reduction depends on NADH level of actively respiring cells forming a nontoxic, aqueous formazan (11). PMS and XTT are widely used in combination as electron carrier and acceptor, respectively, in a colorimetric viability assay of diaphorase-catalyzed redox cycling (12), which indicates the cellular redox potential $(8,11)$.

Hormesis extends life span through the induction of mild redox stress (13), which enhances energy metabolism, activates antioxidant mechanisms, and suppresses reactive oxygen species production (14-17). Nutrient deprivation can be a stressor per se due to 
normal caloric restriction and results in extended longevity (18). The level of nutrition is associated with the redox state (14), and this restriction may cause cellular electron deprivation, impaired mitochondrial function, and suppressed NADH availability (19). In our present study, we investigated whether the longevity of a bdelloid rotifer, Philodina acuticornis, is affected by modulation of its cellular redox state by means of chemical manipulation and extreme caloric restriction $(\mathrm{ECR})$.

\section{Materials and Methods}

In Vivo Invertebrate Model

Our work was performed on invertebrate bdelloid rotifer $P$ acuticornis; therefore, according to the current ethical regulations, no specific ethical permission was needed. The $P$ acuticornis was obtained from Hungarian aquavaristique. Our experiments were carried out in accordance with globally accepted norms: Animals (Scientific Procedures) Act, 1986, associated guidelines, EU Directive 2010/63/ EU for animal experiments, and the National Institutes of Health guide for the care and use of laboratory animals (NIH Publications No. 8023, revised 1978). Animal studies comply with the ARRIVE guidelines. The culturing (eg, standard medium), harvesting, and monitoring methods of $P$ acuticornis have been reported in detail in our prior publication (5).

\section{Treatment Protocols of Rotifer Preselection and Conditioning}

The treatment period (Figure 1A) consisted of two distinct phases: (a) Preselection phase (Figure 1B): Rotifer populations (100 \pm 5 individual/well) and middle-aged (15 days old) one-housed animals (one rotifer/well) were administered PMS ( $5 \mu \mathrm{M}$; P9625, Sigma-Aldrich, St. Louis, MO) combined with XTT $(1,000 \mu \mathrm{M}$; X4626, SigmaAldrich) under total food deprivation for 72 hours in darkness. As a parallel control for XTT, ascorbic acid (AA; 1,000 $\mu \mathrm{M}$; A1300000, Sigma-Aldrich) was applied. (b) Conditioning phase (Figure $1 \mathrm{C}$ and D): First, the dead animals and the remnants of the treatment compounds were eliminated from the wells by washing. The selected surviving individuals were treated permanently (with the medium changed in every second day) with $50 \mu \mathrm{M}$ XTT or AA (corresponding to their respective preselection treatment; $n=90$ ) under standard feeding condition (Figure 1C; $600 \mu \mathrm{g} / \mathrm{mL}$ ) or ECR (Figure 1D; $50 \mu \mathrm{g}$ / $\mathrm{mL}$ ). Survival of rotifers related to different amount of food was also measured (Supplementary Method and Supplementary Figure 1A). The number of live rotifers, endogenic NADH, and the mastax contraction frequency were used to assess treatment effects. The intracellular NADH levels (Figure 1B; $n=10$ well/group) were measured (absorbance: $450 \mathrm{~nm}$ ) with NADH Quantification Kit (K337-100, BioVision, Milpitas, CA) following the protocol of the manufacturer and normalized exclusively to survivals of preselection.

\section{Characterization of Rotifers With Different Viability Markers}

The representative photographs were taken by Leitz Labovert FS microscope combined with DSLR camera (Nikon D5500, Nikon Corp, Japan) in $\times 400$ magnification. To characterize and compare middle-aged normal (NRs; naïe, 15 days old; length: $285 \mu \mathrm{m}$, width: $57 \mu \mathrm{m}$ ) and senescent super rotifers (SRs; preselected with PMS/XTT/total food deprivation and subsequently conditioned with XTT under ECR until 110 days of age; length: $393 \mu \mathrm{m}$, width: $108 \mu \mathrm{m}$ ), the following markers (Figure 2B) were used at end-point

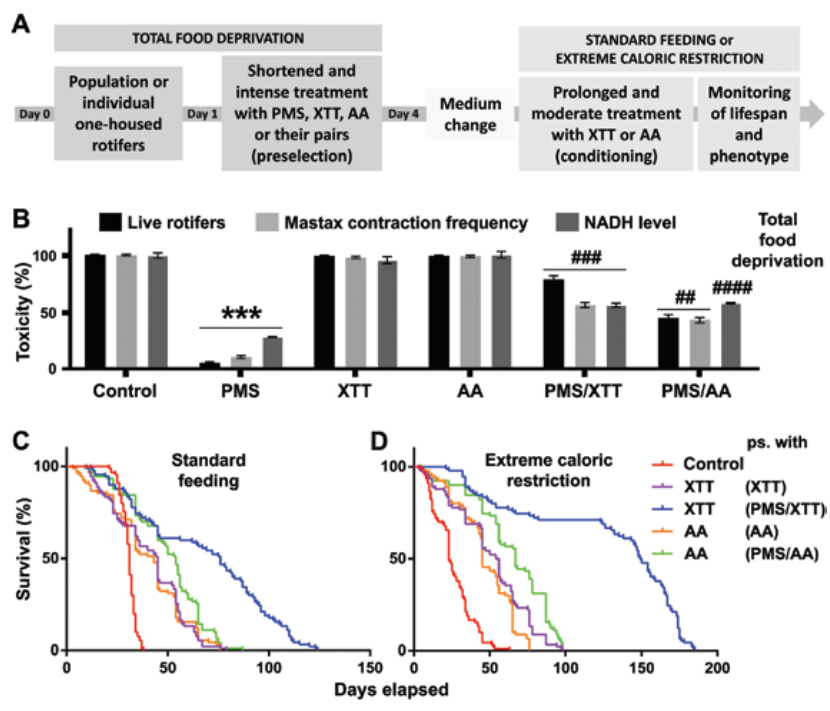

Figure 1. The effect of redox state modulation on the survival and phenotype of rotifers. Panel A presents the schematic work flow of the redox modulating protocol (A). The intense chemical electron deprivation in the preselection phase caused significant toxicity under total food deprivation (B). The error bars present SEM. One-way analysis of variance with Bonferroni post hoc test was used for statistical analysis, the levels of significance were "\#p $p$ $.01,{ }^{* * * * \# \#} p<.001$, and ${ }^{\# \# \#} p<.0001{ }^{*}$, significant difference from untreated controls; \#, significant difference from the group receiving PMS alone). Treatment of preselected surviving rotifers with moderate chemical electron deprivation (XTT or AA) under standard feeding conditions (C) or under extreme caloric restriction (D) significantly extended their life span. KaplanMeier survival curves are presented ( $n=90$, one-housed rotifer per dose of agent indicated). Abbreviations: $A A=$ ascorbic acid; $P M S=$ phenazine methosulfate.

monitoring (after applying standard feeding for a 10-day period with no further chemical treatment): mastax contraction frequency, body size index, viable egg production $(n=35-35$ for all features in both groups, one-housed individuals), and the number of descendants in the population $(n=10-10$, flasks; started from 5-5 NR or SR animals per each). Freezing tolerance was also compared between the phenotypes. Ten-ten flasks (media fully decanted) of NR and SR $(2,000 \pm 100$ individuals per flask) were frozen for 24 hours at $-75^{\circ} \mathrm{C}$. The number of survivors was counted 24 hours after thawing (using $20 \mathrm{~mL}$ media per flasks at room temperature).

\section{Statistics}

Statistical analysis was performed with SPSS 23.0 (SPSS Inc, Chicago, IL) using one-way analysis of variance with Bonferroni post hoc test. The data were presented in mean $\pm S E M$; the levels of significance were ${ }^{*, \#} p<.05 ; * * \# p<.01 ; * * * \# \# p<.001 ; \# \# \# p<.0001$. KaplanMeier curves were applied to present the survival of the groups. The GraphPad Prism 7.0b software (GraphPad Software Inc, La Jolla, CA) was used for the illustration and statistical analysis of survival (log-rank; Mantel-Cox).

\section{Results and Discussion}

In the rotifers, the electron source was their own plasma membrane redox system. This apparatus supposedly attenuates the aging-associated oxidative stress by assuring $\mathrm{NAD}^{+}$for ATP-producing biological oxidation (20). Intracellular NADH is the reducing source for extracellular XTT reduction, providing electrons via plasma 

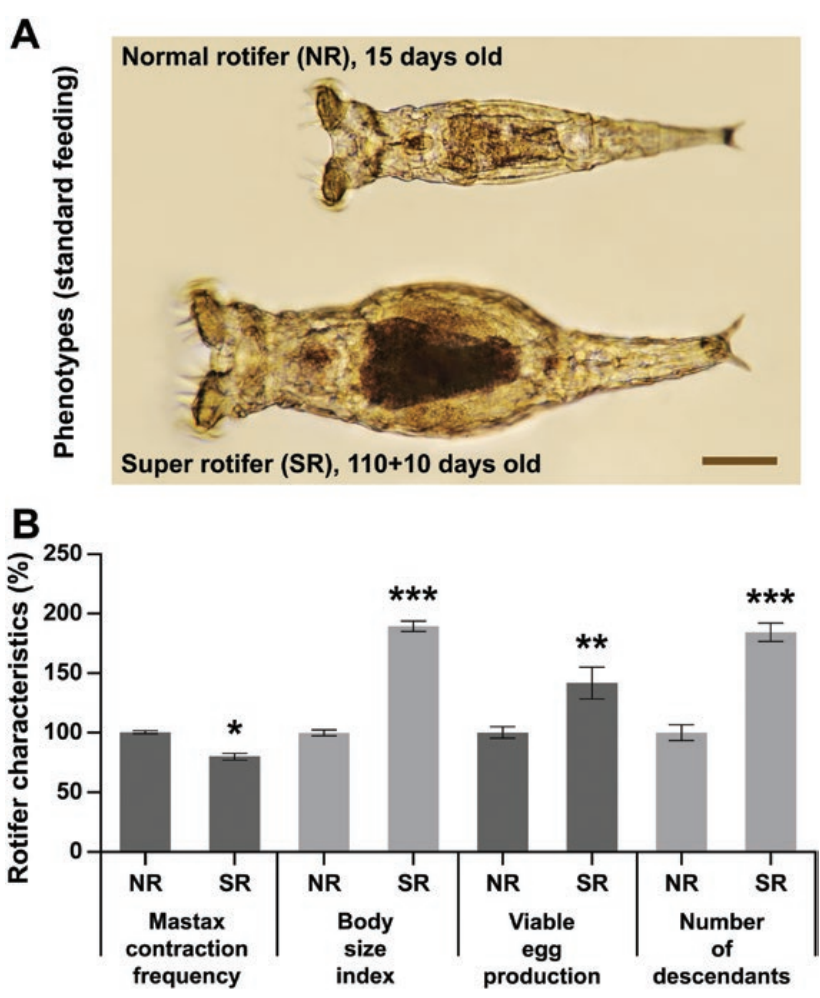

Figure 2. Characterization and comparison of middle-aged normal rotifers (NRs; naïve, 15 days old) and senescent super rotifers (SRs; 110 days old) after a 10-day period of standard feeding without any chemical treatment. A representative illustration of the difference in body size between NR and SR is provided (A; scale bar: $50 \mu \mathrm{m})$. The viability markers of SR compared with NR specimens significantly changed (B). The error bars present SEM. One-way analysis of variance with Bonferroni post hoc test was used for statistical analysis; the levels of significance were ${ }^{*} p<.05,{ }^{* *} p<.01$, and ${ }^{* *} p<.001$. $(*$, significant difference from NR within the respective category).

membrane redox system (21). PMS, as an intermediate electron carrier, markedly promotes this reaction. The low redox potential of PMS results electron deprivation in living systems (22). To confirm the reliability of the PMS- and XTT-containing system, we tested this combination (cell-free) in the presence of NADH (Supplementary Figure 1B).

Our in vivo viability experiments consisted of two main treatment phases (Figure 1A) using middle-aged (15 days old) rotifers. First, we preselected individuals by testing the electron deprivation tolerance, by treatment with PMS and XTT combined or alone under total food deprivation for 72 hours (Figure 1B). As a parallel control for XTT, we used AA, a well-known antioxidant (16). The combination of PMS/XTT or PMS/AA resulted in significantly lower decreases in the number of live rotifers and intracellular NADH level compared with PMS treatment alone, as did also regarding mastax contraction frequency, a validated index of rotifer viability (5). Neither XTT nor AA alone had any effect on viability markers. The reduced form of PMS can alone generate reactive oxygen species in a dose-dependent manner (23), and we presume that the protective role of XTT and AA (via facilitation of NADH metabolism) might be secondary to an irreversible capturing of electrons from PMS. The data of live rotifers, mastax contraction frequency, and intracellular $\mathrm{NADH}$ level show good correlation (Figure 1B).

The surviving rotifers were subjected to the next phase (Figure 1C and D) of experiments. This period was associated with a permanent (for several months) and moderate (low dose) chemical electron deprivation accompanied by standard feeding or ECR. The survival of $P$ acuticornis was measured under various treatment conditions applying standard feeding (Figure 1C) or ECR (Figure 1D). Extreme low nutrition alone is capable of triggering electron deprivation $(17,18)$. However, when ECR was combined with XTT treatment $(50 \mu \mathrm{M})$ in preselected (PMS/XTT/total food deprivation preconditioned) rotifers, it synergistically extended the life span to an extreme extent (up to 182 days) compared with untreated-untreated controls (Figure 1D). The respective treated-untreated controls underwent chemical/dietary preselection, but not receiving chemical treatment in the second phase (data not shown). Less pronounced but likewise outstanding longevity was observed in the corresponding XTTtreated group (PMS/XTT/total food deprivation preconditioned) under standard feeding (Figure 1C) during second phase and (to a lesser extent) the corresponding AA-treated groups (PMS/AA/total food deprivation preconditioned) with a slight superiority of ECR (Figure 1D) over standard feeding. XTT and AA both significantly (though less remarkably) increased the life span of preconditioned rotifers compared with untreated-untreated controls (Figure 1C and D) and the respective treated-untreated controls (data not shown), with no remarkable influence of diet.

The combination of PMS/XTT (under total food deprivation) preselection followed by XTT (under ECR) made a synergistic positive impact on life span and phenotype of preselection survivors resulting in what we call super rotifers, a "nickname" reflecting their collection of unique properties (see below). The phenomenon of this markedly prolonged life span, related to $P$ acuticornis, has not been previously published. The documented maximal life span was approximately 75 days, with mean 43 days (24).

In our another experiment, middle-aged ( 15 days old) NRs and senescent SRs (110 days old) were followed up for 10 days under standard feeding conditions without any chemical treatment to compare their viability markers (Figure 2). There were significant differences in the phenotype (Figure 2A). First, the "body size index" of SRs was higher than that of NRs. Interestingly, the mastax contraction frequency (5) of SRs was lower compared with NRs, probably as a result of altered neuromuscular function due to increased size. More surprisingly, these senescent (110 days old) animals were capable of laying viable eggs (reflecting an effect on the reproduction phase); moreover, the numbers of offspring were significantly higher after the 10-day period than in NRs (Figure 2B). To our knowledge, no similar long reproductive phase without dormant stage has ever been detected in bdelloids.

Furthermore, we assessed how SRs tolerate freezing $\left(-75^{\circ} \mathrm{C}\right)$, monitoring the recovery rate of cultures 24 hours after being thawed. Starting from $2,000 \pm 100$ individuals per flask $(n=10)$, we found significantly more survivors in the SR $(5.0 \pm 0.52$ individuals $)$ than in NR cultures ( $2.6 \pm 0.40$ individuals).

After reintroduction of standard feeding and thus cessation of permanent conditioning (with ECR and low-dose XTT treatment) at 110 days of age, the "remaining lifetime" of SRs was restored near to their original expected longevity $(25 \pm 4.2$ days $)$ until their natural death.

The developed life-span- and phenotype-modifying system can be a reliable experimental model providing new insights into the processes of senescence. The intracellular NADH regulation is an essential part of this complex mechanism. As a consequence of caloric restriction in bdelloid rotifers with extended life span, the volume of stomach syncytium and vitellarium reduced. The observed physiological changes are accompanied with slowed metabolic 
activity and cease of reproduction (3). Extended lifetime together with the delayed aging is a unique and unprecedented phenotype in the academic literature for bdelloid rotifers.

\section{Supplementary Material}

Supplementary data are available at The Journals of Gerontology, Series A: Biological Sciences and Medical Sciences online.

\section{Funding}

This research did not receive any specific grant from funding agencies in the public, commercial, or not-for-profit sectors. This work was supported by the Australian National Health and Medical Research Council to A.B.

\section{Acknowledgments}

We thank Anna Szentgyorgyi MA and Levente Szalardy MD, PhD for the contribution of in proofreading the manuscript.

\section{Conflict of Interest}

A.B. is a shareholder in Prana Biotechnology Ltd, Cogstate Ltd, Brighton Biotech LLC, Grunbiotics Pty Ltd, Eucalyptus Pty Ltd, and Mesoblast Ltd. $\mathrm{He}$ is a paid consultant for and has a profit share interest in Collaborative Medicinal Development Pty Ltd.

\section{References}

1. Enesco HE. Rotifers in aging research: use of rotifers to test various theories of aging. Hydrobiologia. 1993;255-256:59-70. doi:10.1007/ BF00025821

2. Snare DJ, Fields AM, Snell TW, Kubanek J. Lifespan extension of rotifers by treatment with red algal extracts. Exp Gerontol. 2013;48:1420-1427. doi:10.1016/j.exger.2013.09.007

3. Marotta R, Uggetti A, Ricci C, Leasi F, Melone G. Surviving starvation: changes accompanying starvation tolerance in a bdelloid rotifer. $J$ Morphol. 2012;273:1-7. doi:10.1002/jmor.11000

4. Ricci C. Culturing of some bdelloid rotifers. Hydrobiologia. 1984;122:4551. doi:10.1007/BF00007665

5. Olah Z, Bush AI, Aleksza D, et al. Novel in vivo experimental viability assays with high sensitivity and throughput capacity using a bdelloid rotifer. Ecotoxicol Environ Saf. 2017;144:115-122. doi:10.1016/j. ecoenv.2017.06.005

6. Lin SJ, Ford E, Haigis M, Liszt G, Guarente L. Calorie restriction extends yeast life span by lowering the level of NADH. Genes Dev. 2004;18:1216. doi:10.1101/gad.1164804

7. Marcu R, Wiczer BM, Neeley CK, Hawkins BJ. Mitochondrial matrix $\mathrm{Ca}^{2+}$ accumulation regulates cytosolic $\mathrm{NAD}^{+} / \mathrm{NADH}$ metabolism, protein acetylation, and sirtuin expression. Mol Cell Biol. 2014;34:2890-2902. doi:10.1128/MCB.00068-14

8. Berridge MV, Herst PM, Tan AS. Tetrazolium dyes as tools in cell biology: new insights into their cellular reduction. Biotechnol Annu Rev. 2005;11:127-152. doi:10.1016/\$1387-2656(05)11004-7
9. Paull KD, Shoemaker RH, Boyd MR, et al. The synthesis of XTT: a new tetrazolium reagent that is bioreducible to a water-soluble formazan. $J$ Heterocycl Chem. 1988;25:911-914. doi:10.1002/jhet.5570250340

10. Scudiero DA, Shoemaker RH, Paull KD, et al. Evaluation of a soluble tetrazolium/formazan assay for cell growth and drug sensitivity in culture using human and other tumor cell lines. Cancer Res. 1988;48:4827-4833.

11. Comley JC, Turner CH. Potential of a soluble tetrazolium/formazan assay for the evaluation of filarial viability. Int J Parasitol. 1990;20:251-255. doi:10.1016/0020-7519(90)90107-X

12. Halaka FG, Babcock GT, Dye JL. Properties of 5-methylphenazinium methyl sulfate. Reaction of the oxidized form with NADH and of the reduced form with oxygen. J Biol Chem. 1982;257:1458-1461.

13. Cypser JR, Johnson TE. Multiple stressors in Caenorhabditis elegans induce stress hormesis and extended longevity. J Gerontol A Biol Sci Med Sci. 2002;57:B109-B114. doi:10.1093/gerona/57.3.B109

14. Hyun DH, Emerson SS, Jo DG, Mattson MP, de Cabo R. Calorie restriction up-regulates the plasma membrane redox system in brain cells and suppresses oxidative stress during aging. Proc Natl Acad Sci USA. 2006;103:19908-19912. doi:10.1073/pnas.0608008103

15. Rea SL, Ventura N, Johnson TE. Relationship between mitochondrial electron transport chain dysfunction, development, and life extension in Caenorhabditis elegans. PLoS Biol. 2007;5:e259. doi:10.1371/journal. pbio.0050259

16. Szalárdy L, Zádori D, Klivényi P, Toldi J, Vécsei L. Electron transport disturbances and neurodegeneration: from Albert Szent-Györgyi's concept (szeged) till novel approaches to boost mitochondrial bioenergetics. Oxid Med Cell Longev. 2015;2015:498401. doi:10.1155/2015/498401

17. Weithoff G. Dietary restriction in two rotifer species: the effect of the length of food deprivation on life span and reproduction. Oecologia. 2007;153:303-308. doi:10.1007/s00442-007-0739-6

18. Gribble KE, Kaido O, Jarvis G, Mark Welch DB. Patterns of intraspecific variability in the response to caloric restriction. Exp Gerontol. 2014;51:28-37. doi:10.1016/j.exger.2013.12.005

19. Chandrasekaran A, Idelchik MDPS, Melendez JA. Redox control of senescence and age-related disease. Redox Biol. 2017;11:91-102. doi:10.1016/j. redox.2016.11.005

20. Hyun DH, Hernandez JO, Mattson MP, de Cabo R. The plasma membrane redox system in aging. Ageing Res Rev. 2006;5:209-220. doi:10.1016/j. arr.2006.03.005

21. McCluskey C, Quinn JP, McGrath JW. An evaluation of three newgeneration tetrazolium salts for the measurement of respiratory activity in activated sludge microorganisms. Microb Ecol. 2005;49:379-387. doi:10.1007/s00248-004-0012-z.

22. Kettisen K, Bülow L, Sakai H. Potential electron mediators to extract electron energies of RBC glycolysis for prolonged in vivo functional lifetime of hemoglobin vesicles. Bioconjug Chem. 2015;26:746-754. doi:10.1021/ acs.bioconjchem. 5 b00076

23. Gabriel JE, Guerra-Slompo EP, de Souza EM, de Carvalho FA, Madeira HM, de Vasconcelos AT. Superoxide radical-generating compounds activate a predicted promoter site for paraquat-inducible genes of the Chromobacterium violaceum bacterium in a dose-dependent manner. Genet Mol Res. 2015;14:10139-10144. doi:10.4238/2015. August.21.20

24. Ricci C, Perletti F. Starve and survive: stress tolerance and lifehistory traits of a bdelloid rotifer. Funct Ecol. 2006;20:340-346. doi:10.1111/j.1365-2435.2006.01082.x 